Keywords: Schizophrenia; Perineuronal oligodendrocytes; Inferior parietal lobule; Insight; Morphometry.

\title{
Deficit of perineuronal oligodendrocytes in the inferior parietal lobule is associated with lack of insight in schizophrenia
}

\author{
Victor M. Vostrikov \\ Natalya S. Kolomeets \\ Natalya A. Uranova
}

Laboratory of Clinical Neuropathology, Mental Health Research Center, Moscow

RUSSIA

\begin{abstract}
Background and Objectives: Previously we reported a significant reduction in the numerical density of oligodendrocytes and oligodendrocyte clusters in the inferior parietal lobule (IPL) in schizophrenia that was associated with lack of insight. We also found a significant decrease in the number of perineuronal oligodendrocytes (PnOl) in the prefrontal cortex in schizophrenia and therefore we hypothesized that there may also be a deficit of $\mathrm{PnOl}$ in the IPL in schizophrenia and that it could be associated with poor insight.

Methods: We estimated the number of PnOl adjacent to pyramidal neurons in layer 3 of BA39 and BA40 in Nissl stained sections from 24 males with schizophrenia and 24 normal male controls from the Stanley Parietal Collection. The schizophrenia group was divided into three subgroups based on level of insight: poor, fair or good.

Results: We found a significant deficit of PnOl in layer 3 of BA39 and BA40 in the schizophrenia group as compared to the control group $(\mathrm{p}<0.01)$. In the control group but not in the schizophrenia group in BA39 the number of PnOl was significantly higher in the left hemisphere compared to the right hemisphere. In schizophrenia, in BA39 the number of $\mathrm{PnOl}$ was decreased in the subgroup with poor insight vs. controls. In BA40 the subgroups with both poor and fair insight were decreased vs. controls $(\mathrm{p}<0.01)$. In BA40 the subjects with fair insight also differed from those with good insight $(\mathrm{p}<0.01)$.

Conclusions: The reduction of $\mathrm{PnOl}$ in the IPL in schizophrenia is associated with impaired insight and lack of hemispheric asymmetry.
\end{abstract}

Received: 24 December 2013

Revised: 20 March 2014

Accepted: 22 March 2014 


\section{Introduction}

Neuroimaging, genetic and postmortem studies have all provided evidence that a dysfunction and deficits of oligodendrocytes may be involved in further abnormalities of neuronal connectivity in schizophrenia ${ }^{1,2}$ and may also contribute to the various clinical symptoms of schizophrenia including impaired insight into their disorder ${ }^{3-7}$. Impaired insight is a core feature of schizophrenia and an important predictor of functional outcome, prognosis, and treatment adherence ${ }^{8}$. Impaired insight is associated with dysfunction of specific brain structures, including the prefrontal cortex (PFC) as well as the IPL in particular $^{9,10}$. Poor insight is associated with deficient prefrontal cognitive functioning, lower prefrontal volumes and reduced gray matter volume in the IPL in schizophrenia patients ${ }^{10}$. Previously we reported a significant $15 \%$ reduction in the numerical density of oligodendrocytes $^{6}$ and $25 \%$ reduction in oligodendrocyte clusters ${ }^{7}$ in the IPL in schizophrenia and the association of these measures with the lack of insight.

The IPL is among the most highly lateralized areas of the brain, and both decreased cerebral lateralization and reversed asymmetry of the IPL have been reported in schizophrenia $^{10-13}$. A significant lateralization of oligodendrocyte density was detected in layer 3 of the IPL (BA39) (L>R) in the control group but not in the schizophrenia group ${ }^{6}$.

The IPL and the PFC have extensive interconnections and common cortical and subcortical target regions ${ }^{14}$. Previously we reported a reduction in the number of $\mathrm{PnOl}$ in layer 3 of the dorsolateral PFC in schizophrenia ${ }^{15}$. Based on these findings, we hypothesized that a deficit of PnOl may also occur in the IPL in schizophrenia, and that it may be more pronounced in the left hemisphere and in the schizophrenia subjects with poor insight as compared to those with good insight.

The aims of the study were: 1 ) to estimate the number of PnOl in layer 3 of the IPL (BA39, BA40) in schizophrenia and normal controls; 2) to analyze hemispheric effect on the number of $\mathrm{PnOl}$ and 3 ) to study the effect of diagnosis and insight on the number of PnOl.

\section{Materials and Methods}

\section{Samples}

Human brain specimens were donated by the Stanley Medical Research Institute's 'Parietal Collection'. The samples consisted of 48 male subjects ( 24 controls and 24 with schizophrenia). Diagnosis was made according to DSM-IV criteria. A postmortem estimate of each person's awareness of illness (insight) based on the person's clinical records has been previously reported ${ }^{6}$. The mean age at the time of death was $44.3 \pm 9.3$ years for the control group and $39.8 \pm 10.7$ years for the schizophrenia group. The average postmortem interval was $24.4 \pm 10.8$ hours for the control group and 29.1 \pm 11.6 hours for the schizophrenia group. Fourteen cases were from the left hemisphere and ten cases were from the right hemisphere. Tissue of the IPL was available from one hemisphere of each brain. Complete demographic and clinical data were reported in the previous paper ${ }^{6}$.

The brain specimens were coded, and all cytoarchitectural assessments were done blindly. The angular gyrus (BA39) and the supramarginal gyrus (BA40) were identified according to macroscopic landmarks ${ }^{16}$. Ten serial sections through the IPL (every 17th section) were mounted on slides and Nissl-stained. 


\section{Morphometric analysis}

The sublayers of layer 3 ( $a, b$ and $c$ ) in BA39 and BA40 were easily identified based on cytoarchitectural features. The number of PnOl was counted in each sublayer of layer 3 in BA39 and BA40. PnO1 were defined as all oligodendrocytes located within $<5 \mu \mathrm{m}$ of pyramidal neurons. Pyramidal neurons were identified by their triangular shape, oval shaped clear nucleus with a prominent nucleolus, presence of intensively stained cytoplasm and vertical apical dendrite. Oligodendrocytes were identified by the presence of a small round or oval nucleus, with relatively dense nuclear staining (more chromophilic than astroglial nuclei) and a narrow unstained rim of cytoplasm. One hundred pyramidal neurons with identifiable nucleolus were systematically randomly sampled for each sublayer. The number of pyramidal cells sampled from each subject was 300, from each group 7200 , total 14,400 . The number of $\mathrm{PnOl}$ was expressed as the number of oligodendrocytes per neuron.

\section{Statistical analyses}

Statistical analysis was performed using Statistica 7. The data were examined using the Kolmogorov-Smirnov test for normality. A Pearson correlation analysis was performed to assess possible correlations between the parameters measured and age, postmortem interval, $\mathrm{pH}$, refrigerator interval, brain weight, total lifetime antipsychotics, age at onset and duration of disease. Comparisons between patients with schizophrenia and controls were performed using two-way ANOVA with the number of PnOl in three sublayers of layer 3 as the dependent variables, and diagnosis and hemispheres as the independent variables. A one-way ANOVA followed by post hoc Duncan's test was used to compare the control group and the three insight schizophrenia subgroups. A correlation analysis was also performed to assess possible correlations between the number of $\mathrm{PnOl}$ and the numerical density of oligodendrocyte clusters as estimated previously in the same sections ${ }^{7}$.

\section{Results}

\section{Effects of disease and hemispheres}

The mean numbers of $\mathrm{PnOl}$ and the results of their comparisons between the schizophrenia and the control groups for each sublayer of layer 3 are shown in Fig. 1. The ANOVA revealed a significant effect of diagnosis on the number of $\mathrm{PnOl}$ in all sublayers in BA39 $[\mathrm{F}(3,44)=4.57, \mathrm{p}=0.007]$ and in all sublayers in BA40 $[\mathrm{F}(3,44)=3.71, \mathrm{p}=$ 0.018]. A decrease in the mean values of the parameter was found in BA39 in sublayer $3 a(-21 \%, p=0.004)$, in sublayer $3 b(-20 \%$, $\mathrm{p}=0.013)$, in sublayer $3 \mathrm{c}(-24 \%, \mathrm{p}=0.004)$ and in BA40 in sublayer $3 \mathrm{a}(-13 \%, \mathrm{p}=$ $0.059)$, in sublayer $3 b(-16 \%, p=0.007)$ and in sublayer $3 c(-18 \%, p=0.003)$ in the schizophrenia group as compared to the control group. Correlation analysis did not reveal significant effects of postmortem interval, refrigerator interval, brain weight, brain $\mathrm{pH}$, age at onset or duration of disease on the number of $\mathrm{PnOl}$ in BA 39 and BA 40.

There was a significant hemispheric effect on the number of $\mathrm{PnOl}$ in BA39 in layer $3[\mathrm{~F}(3,43)=2.89, \mathrm{p}=0.046]$. A post hoc test demonstrated a significant hemispheric difference in the control group but not in the schizophrenia group: the number of $\mathrm{PnOl}$ was significantly higher in the left hemisphere as compared to the right hemisphere in $3 \mathrm{a}(23 \%), 3 \mathrm{~b}(25 \%)$ and in $3 \mathrm{c}(23 \%)$. There were no significant hemispheric differences in BA 40 (Table 1, Fig. 2). 

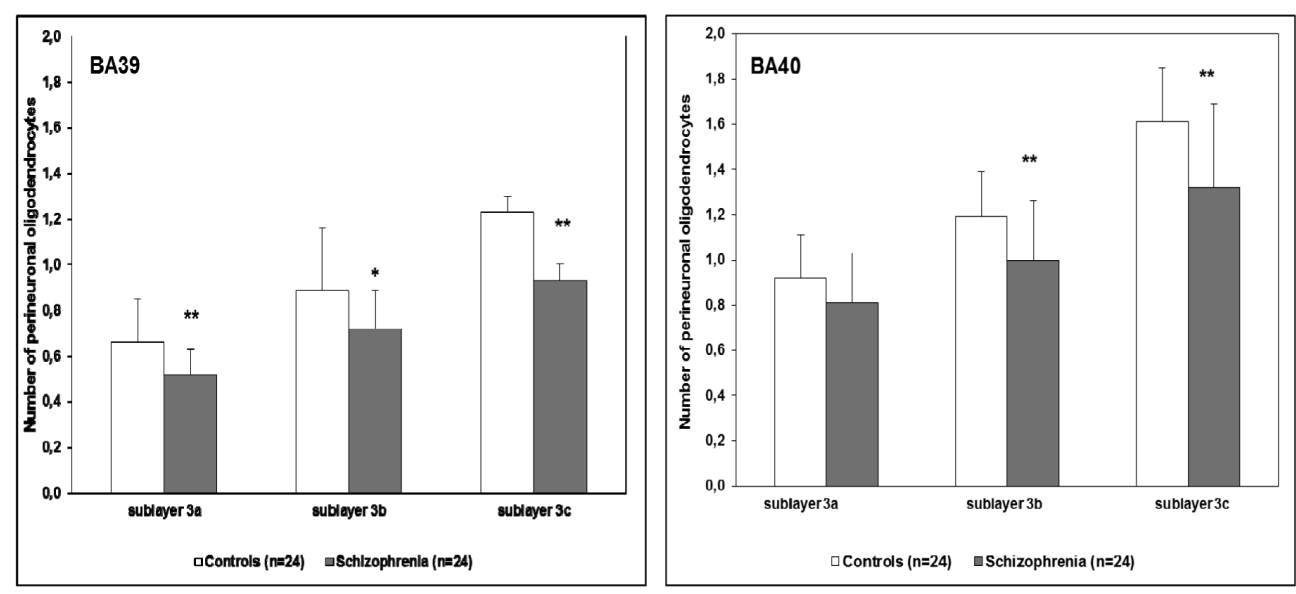

Figure 1. The number of perineuronal oligodendrocytes in three sublayers of layer 3 in BA39 and BA40 in the control group and in the schizophrenia group. p-values: ${ }^{*} \mathrm{p}<0.05, * * \mathrm{p}<0.01$.

Table 1

The number of perineuronal oligodendrocytes (means $\pm \mathrm{SD}$ ), hemispheric effect

\begin{tabular}{llllllr} 
Sublayers & Controls & \multicolumn{5}{l}{ Schizophrenia } \\
\hline & $\begin{array}{l}\text { Left hemisphere } \\
(\mathrm{n}=14)\end{array}$ & $\begin{array}{l}\text { Right hemisphere } \\
(\mathrm{n}=10)\end{array}$ & $\mathrm{p}$ & $\begin{array}{l}\text { Left hemisphere } \\
(\mathrm{n}=14)\end{array}$ & $\begin{array}{l}\text { Right hemisphere } \\
(\mathrm{n}=10)\end{array}$ & $\mathrm{p}$ \\
\hline BA 39 & & & & & \\
\hline 3a & $0.73 \pm 0.17$ & $0.56 \pm 0.17$ & 0.024 & $0.54 \pm 0.08$ & $0.51 \pm 0.15$ & 0.6 \\
3b & $0.98 \pm 0.21$ & $0.74 \pm 0.29$ & 0.03 & $0.73 \pm 0.14$ & $0.69 \pm 0.21$ & 0.5 \\
3c & $1.36 \pm 0.35$ & $1.05 \pm 0.28$ & 0.03 & $0.97 \pm 0.23$ & $0.88 \pm 0.44$ & 0.5 \\
\hline BA 40 & & & & & & \\
\hline 3a & $0.92 \pm 0.23$ & $0.92 \pm 0.11$ & 0.9 & $0.79 \pm 0.22$ & $0.82 \pm 0.25$ & 0.7 \\
3b & $1.15 \pm 0.23$ & $1.24 \pm 0.13$ & 0.4 & $0.99 \pm 0.26$ & $1.02 \pm 0.26$ & 0.7 \\
3c & $1.53 \pm 0.25$ & $1.72 \pm 0.19$ & 0.16 & $1.32 \pm 0.39$ & $1.33 \pm 0.35$ & 0.9 \\
\hline
\end{tabular}

\section{Effects of insight}

There was a significant effect of insight on the number of PnOl in both BA39 and BA40. In BA 39 the subgroup with poor insight had a significantly lower number of $\mathrm{PnOl}$ in each of three sublayers of layer $3[23-33 \%, F(3,44)$ $\geq 3.0, \mathrm{p}<0.02]$ as compared to the control group (Fig. 3) but there were no significant differences between the three insight subgroups. In BA 40 the number of $\mathrm{PnOl}$ in the subgroups of subjects with both poor insight and fair insight were significantly lower than in the control group $(\mathrm{p}<0.02)$. The subjects with fair insight also had significantly lower number of $\mathrm{PnOl}$ than subjects with good insight $(\mathrm{p}<0.02)$ (Fig. 3). Comparing the results of this study with the results of our previous 


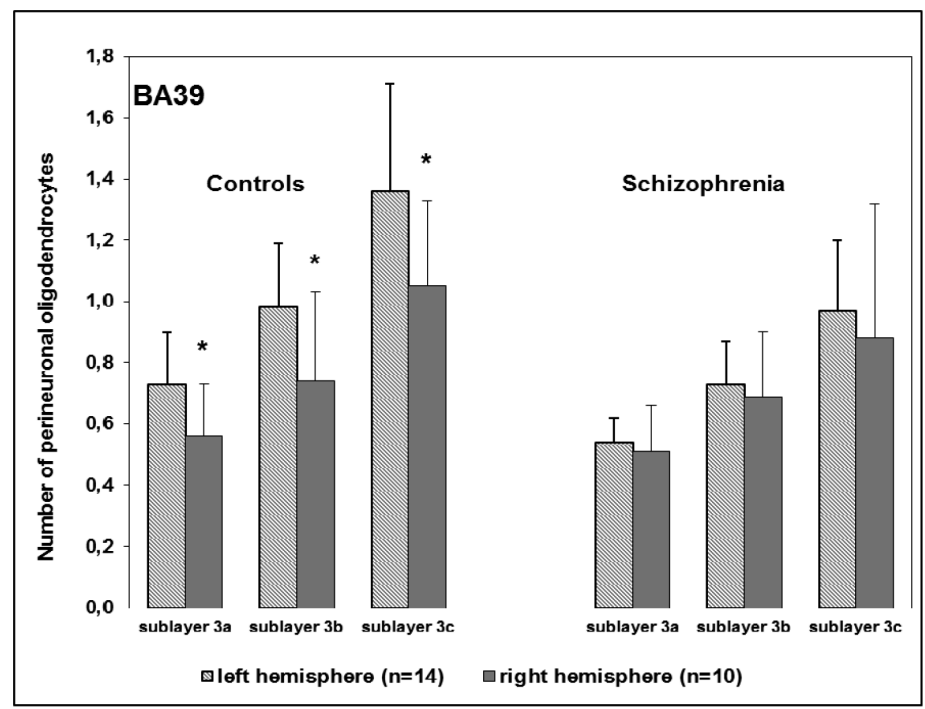

Figure 2. Hemispheric differences in the number of perineuronal oligodendrocytes in three sublayers of layer 3 of BA39 in the control group are absent in the schizophrenia group. p-values: $* \mathrm{p}<0.05$.

Table 2

The number of perineuronal oligodendrocytes (means \pm SD), effect of insight

\begin{tabular}{lcccccc} 
Sublayers & $\begin{array}{c}\text { Controls } \\
(\mathrm{n}=24)\end{array}$ & $\begin{array}{c}\text { Poor insight } \\
(\mathrm{n}=10)\end{array}$ & $\begin{array}{c}\text { Fair insight } \\
(\mathrm{n}=5)\end{array}$ & $\begin{array}{c}\text { Good insight } \\
(\mathrm{n}=9)\end{array}$ & $\mathrm{F}(3.44)$ & $\mathrm{p}$ \\
\hline BA39 & & & & & & \\
\hline 3a & $0.66 \pm 0.19$ & $0.46 \pm 0.09$ & $0.54 \pm 0.07$ & $0.59 \pm 0.12$ & 4.25 & 0.01 \\
$\mathrm{3b}$ & $0.89 \pm 0.27$ & $0.62 \pm 0.14$ & $0.74 \pm 0.21$ & $0.81 \pm 0.13$ & 3.59 & 0.02 \\
\hline 3c & $1.24 \pm 0.35$ & $0.74 \pm 0.28$ & $1.03 \pm 0.33$ & $1.10 \pm 0.30$ & 5.45 & 0.002 \\
\hline BA40 & & & & & & \\
\hline 3a & $0.92 \pm 0.19$ & $0.81 \pm 0.25$ & $0.63 \pm 0.17$ & $0.89 \pm 0.20$ & 2.99 & 0.04 \\
3b & $1.19 \pm 0.20$ & $0.97 \pm 0.26$ & $0.84 \pm 0.21$ & $1.13 \pm 0.23$ & 4.93 & 0.005 \\
3c & $1.61 \pm 0.24$ & $1.29 \pm 0.37$ & $1.04 \pm 0.22$ & $1.53 \pm 0.33$ & 7.06 & 0.0006 \\
\hline
\end{tabular}

study on these same sections, the number of PnOl correlated significantly with the numerical density of oligodendrocyte clusters in three sublayers of layer 3 in BA39 in both control and schizophrenia groups and in BA40 (sublayer c) in the control group but not in the schizophrenia group (Fig. 4).

\section{Discussion}

\section{Patients versus controls}

We found a significant deficit of $\mathrm{PnOl}$ in all sublayers of layer 3 in both BA39 (-23\%) and BA40 (-17\%) in the schizophrenia group 


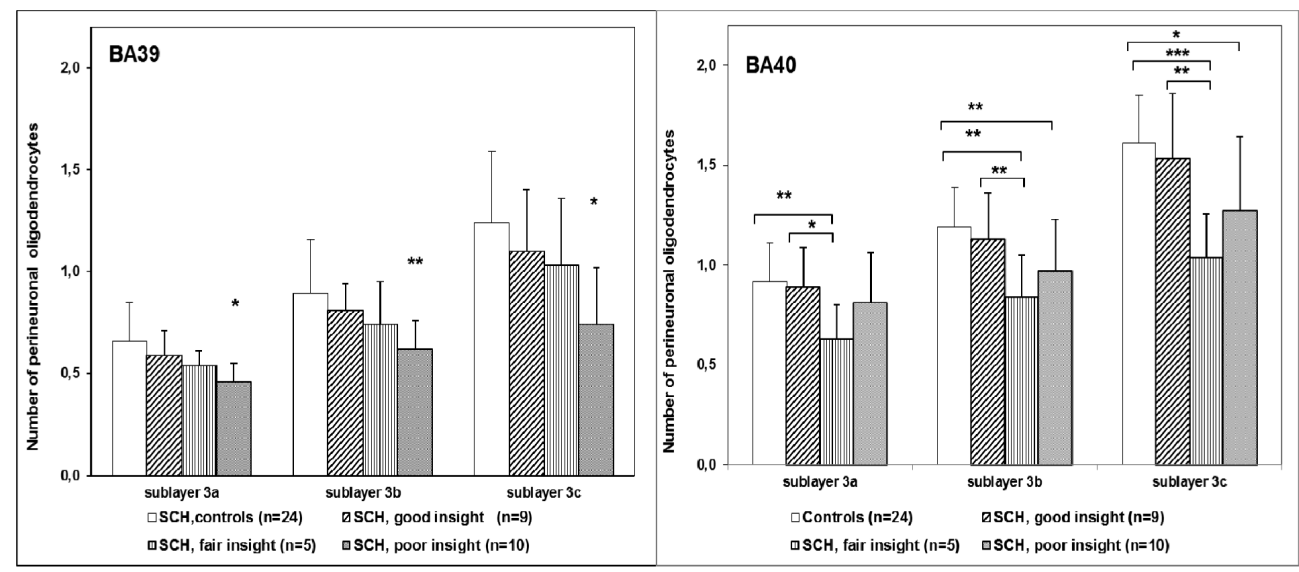

Figure 3. The number of perineuronal oligodendrocytes in the control group and in different insight subgroups in BA39 and BA40. $* \mathrm{p}<0.05, * * \mathrm{p}<0.01, * * * \mathrm{p}<0.001$.

as compared to the control group. The number of PnOl was not correlated with postmortem interval, refrigerator interval, brain weight, brain $\mathrm{pH}$, age at onset or duration of disease in BA 39 and BA 40.

There was no effect of total lifetime antipsychotics on the number of PnOl. However, antipsychotics have been shown to promote the differentiation of oligodendrocyte progenitor cells by regulating oligodendrocyte lineage transcription factors 1 and $2^{17}$. They have also been shown to improve myelin/oligodendrocyte-related dysfunction $^{18}$, and to enhance oligodendrocyte regeneration and myelin repair after cuprizoneinduced demyelination ${ }^{19}$. Together these data would indicate that the decrease in the number of PnOl to not be due to the effects of medication but rather to be associated with the diagnosis of schizophrenia.

We applied a two-dimensional method for the assessment of the number of PnOl. Although this method does not allow for the counting of all oligodendrocytes adjacent to neurons, it does allow for successful comparing of this parameter between the control and disease groups, and our current data con- curs with data previously obtained by the stereological optical dissector method ${ }^{6}$ showing the deficit of the numerical density of oligodendrocytes in layer 3 of the IPL (BA39). The present data are also consistent with the reduced number of $\mathrm{PnOl}$ found in layer 3 of the dorsolateral PFC in schizophrenia ${ }^{15}$, and with the reduction of the numerical density of oligodendrocyte clusters found in the IPL in schizophrenia that was associated with insight in schizophrenia ${ }^{7}$. The findings of this study are also consistent with the results of Byne et $a l .{ }^{20}$ who reported a decrease in the ratio of oligodendrocytes to neurons in the anterior thalamic nucleus in schizophrenia.

Kim and Webster ${ }^{21,22}$ using a genomewide correlation analysis explored the genes and biological processes associated with the number of $\mathrm{PnOl}$ in the PFC in schizophrenia (using the data from our previous study ${ }^{15}$ ). A correlation analysis between genome-wide expression levels and cytoarchitectural traits revealed that 818 genes were significantly correlated with a decrease in the number of $\mathrm{PnOl}$ in schizophrenia subjects as well as in the subjects with bipolar disorder and major depression. Several oligodendrocyte-related 

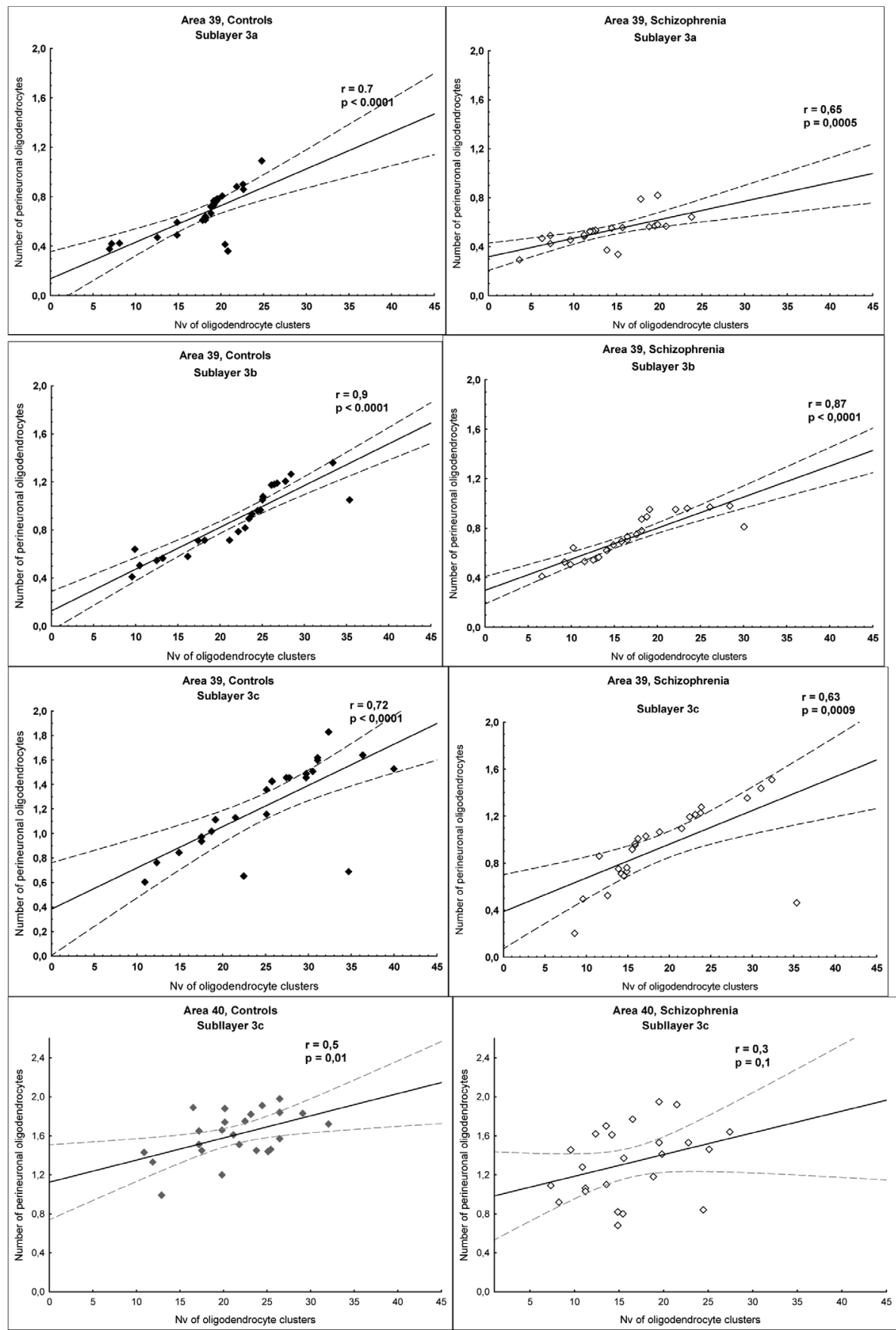

Figure 4. Significant positive correlations between the number of perineuronal oligodendrocytes and the numerical density of oligodendrocyte clusters in BA39 and BA40 in the control group and in the schizophrenia group. 
mRNA, including the mRNA levels for the schizophrenia susceptibility gene ERBB3, showed significant correlations with the number of PnOl. Genome-wide correlation analysis also identified apoptosis as one of the potential mechanisms that may underlie the decrease in the number of $\mathrm{PnOl}$ in the PFC of subjects with schizophrenia. The authors suggest that apoptosis, perhaps associated with cytotoxicity mediated by a glutamate abnormality, may contribute to the reduced numbers of PnOl in the PFC of subjects with major psychiatric disorders. A further analysis ${ }^{23}$ also indicated that several SNPs, including one associated with the glutamine transporter gene SLC38A1 and one with RAB-2A, were associated with the number of $\mathrm{PnOl}$ thus providing novel insights into the possible genetic causalities associated with the deficit of $\mathrm{PnOl}$ in schizophrenia and affective disorders.

\section{Lack of asymmetry of $\mathrm{PnOl}$ in schizophrenia}

The present study showed that the number of PnOl was significantly higher in the left hemisphere than in the right hemisphere in all three sublayers of BA39 in the control group but not in the schizophrenia group. This data is consistent with the results of the previous study that demonstrated a significant lateralization of oligodendrocyte density in layer 3 of the IPL (BA39) (L>R) in the control group but not in the schizophrenia group ${ }^{6}$. The IPL is among the most highly lateralized areas of the brain, and both decreased cerebral lateralization and reversed asymmetry of the IPL, particularly in the angular gyrus, have been reported in schizophrenia ${ }^{10-13}$. Together with the results of the present study, these data provide evidence for the involvement of the oligodendrocytes and of $\mathrm{PnOl}$ in particular in the decreased lateralization of the angular gyrus (BA39) in schizophrenia.

\section{Effects of insight}

We found that in BA39 the number of PnOl was significantly decreased in the schizophrenia subgroup with poor insight as compared to the control group. In BA40 the number of $\mathrm{PnOl}$ was significantly decreased in the subgroups with both poor and fair insight as compared to controls. However, in both areas BA39 and BA40 schizophrenia subjects with poor insight did not differ from subjects with good insight. Although in BA40 the subgroup with fair insight had a significantly lower number of PnOl than subjects with good insight. Thus, the present study shows for the first time that the decreased number of $\mathrm{PnOl}$ in the IPL in schizophrenia is associated with the patient's lack of insight into their disorder. The data are important because the IPL is implicated in neurocognitive impairment in schizophrenia ${ }^{8}$ and cognitive insight can be a useful prognostic indicator, and thus should be considered in future studies and as a potential focus for treatment ${ }^{24}$.

\section{Density of PnOI and oligodendrocyte clustering in schizophrenia}

In BA39 we found decreased number of $\mathrm{PnOl}(-23 \%)$, we previously found that the numerical density of oligodendrocyte clusters was also decreased $(-23 \%)^{7}$. Moreover, in BA39 the number of PnOl correlated significantly with the numerical density of oligodendrocyte clusters in three sublayers of layer 3 in both control and schizophrenia groups. However, in BA40 the correlations were only in the control group but not in the schizophrenia group. Szuchet et al..$^{25}$ in molecular genetic study showed that cortical perineuronal cells are the progeny of oligodendrocyte progenitors and, hence, are member of the oligodendrocyte lineage. Recent experi- 
mental data demonstrated that cell clusters in adult rodent and primate brain contain oligodendrocyte progenitors at different stages of maturation ${ }^{26,27}$.

In addition cell cycle abnormalities and incomplete differentiation of oligodendrocytes have been reported in schizophrenia ${ }^{28-30}$. Together these data suggest that the reduced number of $\mathrm{PnOl}$ in schizophrenia may be associated with the reduced proliferation of oligodendrocyte progenitors. Recent cytochemical and cytological data suggest that $\mathrm{PnOl}$ in the somatosensory cortex support neuronal survival, differentiation, and function, and that they provide protection against neuronal apoptosis (Takasaki et al., 2010) ${ }^{31}$. Thus, the significant decrease in the number of $\mathrm{PnOl}$ may have negative consequence for neuronal activity. In conclusion, the results of the present study suggest that normal oligodendroglia-neuronal relationships in the IPL are likely dysregulated in subjects with schizophrenia primarily due to a decrease of the number of PnOl. The deficit of PnOl is associated with impaired insight and may play a key role in the pathophysiology of schizophrenia. It may also have important implications for the development of treatment strategies.

\section{Authors' contributions}

Dr. Vostrikov designed the study, carried out data collection and interpretation and wrote the manuscript. Dr. Kolomeets contributed to the study design, data collection and interpretation, and preparation of the manuscript. Dr. Uranova designed the study, performed statistical analysis, wrote and revised the manuscript.

\section{Acknowledgments}

The authors would like to thank the Stanley Medical Research Institute for support this work. Postmortem brain sections were donated by Dr. M. J. Webster from the Stanley 'Parietal Collection'. We express our gratitude to Dr. E. Fuller Torrey for examination of clinical records and editing the manuscript and Dr. M.J. Webster for editing the manuscript.

\section{Funding source}

This study was supported by the Stanley Medical Research Institute. Grant number: 07R-1787.

\section{References}

1. Takahashi N, Sakurai T, Davis KL, Buxbaum JD. Linking oligodendrocyte and myelin dysfunction to neurocircuitry abnormalities in schizophrenia. Prog Neurobiol 2011; 93: 13-24.

2. Fields RD. White matter in learning, cognition and psychiatric disorders. Trends in neuroscience 2008; 31: 361-370.

3. Uranova N, Orlovskaya D, Vikhreva O, Zimina I, Kolomeets N, Vostrikov V, et al. Electron microscopy of oligodendroglia in severe mental illness. Brain Res Bull 2001; 55: 597-610.

4. Uranova NA, Vikhreva OV, Rachmanova VI, Orlovskaya DD. Ultrastructural alterations of myelinated fibers and oligodendrocytes in the prefrontal cortex in schizophrenia: A postmortem morphometric study. Schizophr Res and Treatment 2011; 2011: 325789 .

5. Vostrikov V, Orlovskaya D, Uranova N. Deficit of pericapillary oligodendrocytes in the prefrontal cortex in schizophrenia. World J Biol Psychiatr 2008; 9: 34-42.

6. Vostrikov VM, Kolomeets NS, Uranova NA. Reduced oligodendroglial density in neocortex and lack of insight in schizophrenia. Eur J Psychiat 2013; 27: 111-121.

7. Kolomeets NS, Vostrikov VM, Uranova NA. Abnormalities in oligodendrocyte clusters in the inferior parietal cortex in schizophrenia are associated with insight schizophrenia. Eur J Psychiat 2013; 27: 248-258. 
8. van der Meer L, de Vos AE, Stiekema AP, Pijnenborg GH, van Tol MJ, Nolen WA, et al. Insight in schizophrenia: involvement of self-reflection networks? Schizophr Bull 2013; 39: 1288-1295.

9. Tu PC, Lee YC, Chen YS, Li CT, Su TP. Schizophrenia and the brain's control network: aberrant within- and between-network connectivity of the frontoparietal network in schizophrenia. Schizophr Res 2013; 147: 339-347.

10. Torrey EF. Schizophrenia and the inferior parietal lobule. Schizophr Res 2007; 97: 215-225.

11. Smiley JF, Konnova K, Bleiwas C. Cortical thickness, neuron density and size in the inferior parietal lobe in schizophrenia. Schizophr Res 2012; 136: 43-50.

12. Niznikiewicz M, Donnino R, McCarley RW, Nestor PG, Iosifescu DV, O'Donnell B, et al. Abnormal angular gyrus asymmetry in schizophrenia. Am J Psychiatr 2000; 157: 428-437.

13. Swanson N, Eichele T, Pearlson G, Kiehl K, Yu Q, Calhoun VD. Lateral differences in the default mode network in healthy controls and patients with schizophrenia. Hum Brain Mapp 2011; 32: 654-664.

14. Petrides M, Pandya DN. Projections to the frontal cortex from the posterior parietal region in the rhesus monkey. J Comp Neurol 1984; 228: 105-116.

15. Vostrikov VM, Uranova NA, Orlovskaya DD. Deficit of perineuronal oligodendrocytes in the prefrontal cortex in schizophrenia and mood disorders. Schizophr Res 2007; 94: 273-280.

16. Zillles K. Architecture of the human cerebral cortex. In: Paxinos G, Mai JK, eds. The human nervous system. 2nd edition. Elsevier Academic Press; p. 997-1055.

17. Fang F, Zhang H, Zhang Y, Xu H, Huang Q, Adilijiang A, et al. Antipsychotics promote the differentiation of oligodendrocyte progenitor cells by regulating oligodendrocyte lineage transcription factors 1 and 2. Life Sci 2013; 2013; 93: 429-434.

18. Ren Y, Wang H, Xiao L. Improving myelin/oligodendrocyte-related dysfunction: a new mechanism of antipsychotics in the treatment of schizophrenia? Int J Neuropsychopharmacol 2013; 16: 691-700.

19. Zhang Y, Zhang H, Wang L, Jiang W, Xu H, Xiao L, et al. Quetiapine enhances oligodendrocyte regeneration and myelin repair after cuprizone-induced demyelination. Schizophr Res 2012; 138: 8-17.

20. Byne W, Kidkardnee S, Tatusov A, Yiannoulos G, Buchsbaum MS, Haroutunian V. Schizophrenia-associated reduction of neuronal and oligodendrocyte numbers in the anterior principal thalamic nucleus. Schizophr Res 2006; 85: 245-253.

21. Kim S, Webster MJ. Correlation analysis between genome-wide expression profiles and cytoarchitectural abnormalities in the prefrontal cortex of psychiatric disorders. Mol Psychiatry 2010; 15: 326-336.
22. Kim S, Webster MJ. The stanley neuropathology consortium integrative database: a novel, web-based tool for exploring neuropathological markers in psychiatric disorders and the biological processes associated with abnormalities of those markers. Neuropsychopharmacology 2010; 35: 473-482.

23. Kim S, Webster MJ. Integrative genome-wide association analysis of cytoarchitectural abnormalities in the prefrontal cortex of psychiatric disorders. Mol Psychiatry 2011; 16: 452-461.

24. O'Connor JA, Wiffen B, Diforti M, Ferraro L, Joseph C, Kolliakou A, et al. Neuropsychological, clinical and cognitive insight predictors of outcome in a first episode psychosis study. Schizophr Res 2013; 149: 70-76.

25. Szuchet S, Nielsen JA, Lovas G, Domowicz MS, de Velasco JM, Maric D, et al. The genetic signature of perineuronal oligodendrocytes reveals their unique phenotype. Eur J Neurosci 2011; 34: 1906-1922.

26. Kang SH, Fukaya M, Yang JK, Rothstein JD, Bergles DE. NG2+ CNS glial progenitors remain committed to the oligodendrocyte lineage in postnatal life and following neurodegeneration. Neuron 2010; 68: 668-681.

27. Peters A, Sethares C. Oligodendrocytes, their progenitors and other neuroglial cells in the aging primate cerebral cortex. Cerebral Cortex 2004; 14: 995-1007.

28. Radu A, Hristescu G, Katsel P, Haroutunian V, Davis KL. Microarray database mining and cell differentiation defects in schizophrenia. Adv Exp Med Biol 2011; 696: 67-74.

29. Katsel P, Davis KL, Li C, Tan W, Greenstein E, Kleiner Hoffman LB, et al. Abnormal indices of cell cycle activity in schizophrenia and their potential association with oligodendrocytes. Neuropsychopharmacology 2008; 33 : 2993-3009.

30. Kerns DC, Vong GS, Barley K, Dracheva S, Katsel P, Casaccia $\mathrm{P}$, et al. Gene expression abnormalities and oligodendrocyte deficits in the internal capsule in schizophrenia. Schizophr Res 2010; 120: 150-158.

31. Takasaki C, Yamasaki M, Uchigashima M, Konno K, Yanagawa Y, Watanabe M. Cytochemical and cytological properties of perineuronal oligodendrocytes in the mouse cortex. Eur J Neurosci 2010; 32: 1326-1336.

Corresponding author:

Victor M. Vostrikov

Laboratory of Clinical Neuropathology

Mental Health Research Center

Zagorodnoe shosse 2

117152, Moscow, Russia

Tel: +7-495-952-87-70

Fax: +7-495-952-89-40

E-mail: vostrikovvm@mail.ru 